\title{
EXOGENOUS NITRIC OXIDE PREVENTS CARDIOVASCULAR COLLAPSE DURING HEMORRHAGIC SHOCK
}

\author{
Parimala Nachuraju ${ }^{1}$, Adam J. Friedman ${ }^{2}$, Joel M. Friedman ${ }^{1}$, and Pedro Cabrales ${ }^{3}$ \\ ${ }^{1}$ Department of Physiology and Biophysics, Albert Einstein College of Medicine, Bronx, NY \\ ${ }^{2}$ Division of Dermatology, Department of Medicine, Albert Einstein College of Medicine, Bronx, \\ NY \\ ${ }^{3}$ Department of Bioengineering, University of California, San Diego, La Jolla, CA
}

\begin{abstract}
This study investigated the systemic and microvascular hemodynamic changes related to increased nitric oxide (NO) availability following significant hemorrhage, made available by administration of NO releasing nanoparticles (NO-nps). Hemodynamic responses to hemorrhagic shock were studied in the hamster window chamber. Acute hemorrhage was induced by arterial controlled bleeding of 50\% of blood volume, and the resulting hemodynamic parameters were followed over $90 \mathrm{~min}$. Exogenous NO was administered in the form of NO-nps $(5 \mathrm{mg} / \mathrm{kg}$. suspended in $50 \mu 1$ saline) 10 min following induced hemorrhage. Control groups received equal dose of $\mathrm{NO}$ free nanoparticles (Control-nps) and Vehicle solution. Animals treated with NO-nps partially maintained systemic and microvascular function during hypovolemic shock compared to animals treated with Control-nps or the Vehicle ( $50 \mu \mathrm{l}$ saline). The continuous NO released by the NO-nps reverted arteriolar vasoconstriction, partially recovered both functional capillary density and microvascular blood flows. Additionally, NO supplementation post hemorrhage prevented cardiac decompensation, and thereby maintained and stabilized the heart rate. Paradoxically, the peripheral vasodilation induced by the NO-nps did not decrease blood pressure, and combined with NO's effects on vascular resistance, NO-nps promoted intravascular pressure redistribution and blood flow, avoiding tissue ischemia. Therefore, by increasing NO availability with NO-nps during hypovolemic shock, it is possible that cardiac stability and microvascular perfusion can be preserved, ultimately increasing survivability and local tissue viability, and reducing hemorrhagic shock sequelae. The relevance, stability, and efficacy of exogenous NO therapy in the form of NO-nps will potentially facilitate the intended use in battlefield and trauma situations.
\end{abstract}

\section{Keywords}

Microcirculation; hemorrhage; glutathione; nitrosoglutathione; functional capillary density

(C) 2011 Elsevier Ireland Ltd. All rights reserved.

Correspondence to: Pedro Cabrales, Ph.D., University of California, San Diego, Department of Bioengineering, 0412 , 9500 Gilman Dr., La Jolla, CA 92093-0412, Telephone: (858) 534-5847, pcabrales@ucsd.edu.

Publisher's Disclaimer: This is a PDF file of an unedited manuscript that has been accepted for publication. As a service to our customers we are providing this early version of the manuscript. The manuscript will undergo copyediting, typesetting, and review of the resulting proof before it is published in its final citable form. Please note that during the production process errors may be discovered which could affect the content, and all legal disclaimers that apply to the journal pertain.

Conflict of interest statement

No conflicts of interest to declare. 


\section{INTRODUCTION}

Hemorrhagic shock accounts for about $50 \%$ of battlefield deaths in conventional warfare ${ }^{1}$.

Early, aggressive, high volume resuscitation was widely accepted and most practiced during the Vietnam $\mathrm{War}^{2}$. However, metabolic benefits of this approach were only demonstrated in controlled hemorrhage animal models, and when implemented in humans, showed severe deleterious effects including pulmonary failure and re-bleeding ${ }^{3,4}$. Small volume resuscitation can be considered a model to improve the efficiency of fluid therapy by changing the composition and infusion regimens ${ }^{5}$. Even following trauma, blood transfusion produces inflammatory responses, potentially causing more morbidity than the original insult ${ }^{6}$.

Nitric oxide (NO) plays several major roles in human physiology. NO is a neurotransmitter, a macrophage-derived host-defense molecule, an inhibitor of platelet aggregation and endothelium adhesion molecule expression, an antioxidant, cardiac chronotropic, and a potent vasodilator. ${ }^{7}$ Our hypotheses to explain the hemorrhagic shock induced cardiovascular collapse are that the production of $\mathrm{NO}$ is impaired by 1) drastic reduction on endothelial shear stress (due to low blood pressure, cardiac output, circulating volume and blood viscosity ${ }^{8}$; 2) free radical formation/accumulation, uncoupling the endothelial NO synthase (NOS) ${ }^{9}$; and 3 ) hypoxia, as oxygen is a substrate for NO production ${ }^{10}$. Thus, restoration of NO bioavailability impaired during hemorrhagic shock will reduce many hemorrhagic shock induced complications. Since endothelial synthesized NO is the major source of NO for the vasculature ${ }^{11}$, we proposed an intraluminal source, such NO releasing nanoparticles (NO-nps). NO-nps retains NO in a stable form when dry, releases NO upon exposure to moisture, and are stable over long-term dry storage. Unlike most NO generating materials which rely on chemical decomposition or enzymatic catalysis, the NO-nps utilize a thermally driven redox reaction involving nitrite to generate the $\mathrm{NO}^{12,13}$. NO-np half-life

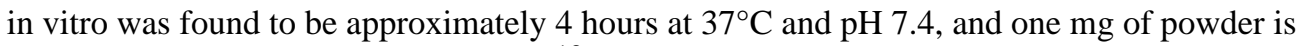
capable of releasing $0.32 \mu$ mols of $\mathrm{NO}^{13}$. When suspended at lower $\mathrm{pH}$ levels, the total mount of NO released did not change, but faster rates of release were measured. In vivo, circulating NO-np produced a decrease in blood pressure, microvascuslar vasodilatation and increased blood flow in a dose dependent fashion. Maximal decrease in pressure in animals treated with NO-np was measured at 90 min after infusion and lasted $3 \mathrm{~h}$ after infusion. Exhaled NO increased after infusion of NO-np, maximal exhaled NO levels were measured $1 \mathrm{~h}$ after infusion and maintained over a period of $2 \mathrm{~h}$; after that exhaled NO decreased. Importantly, one mg of NONOates releases 30 times more than the NO-np ${ }^{13}$.

The objectives of this study were: i) to determine any protective effect of exogenous NO released from NO-nps during hypovolemic shock; ii) to define the basic mechanisms through which exogenous NO restore microvascular function in the absence of volume resuscitation. To achieve these objectives, we subjected our experimental hamster model to a hemorrhage of $50 \%$ of blood volume (BV). Ten minutes following hemorrhage, animals were treated with $5 \mathrm{mg} / \mathrm{kg}$ of NO-nps or NO free nanoparticles (Control-nps), suspended in $50 \mathrm{ul}$ of normal saline. Systemic and microvascular parameters were studied up to $90 \mathrm{~min}$ after hemorrhage.

\section{METHODS}

\section{Synthesis of NO-nps and Control-nps}

The synthesis of NO-nps was recently reported ${ }^{12}$. Briefly, a hydrogel/glass composite was synthesized using a mixture of tetramethylorthosilicate, polyethylene glycol, chitosan, glucose, and sodium nitrite in a $0.5 \mathrm{M}$ sodium phosphate buffer $(\mathrm{pH} 7)$. As previously described, nitrite is reduced to $\mathrm{NO}$ within the matrix as a result of the glasys properties of 
the composite, which allow for redox reactions initiated, with thermally generated electrons from glucose ${ }^{12}$. The composite is dried using a lyophilizer and crushed using planetary ball mill, resulting in nanoparticles containing NO. Once exposed to an aqueous environment, the hydrogel allows for an opening of the water channels inside the particles, facilitating the release of the trapped NO over extended time periods. Control-nps, free of NO inside, were produced identically, but without nitrite.

\section{Animal Preparation}

Investigations were performed in 50 - 65 g male Golden Syrian Hamsters (Charles River Laboratories, Boston, MA) fitted with a dorsal skinfold chamber window. Animal handling and care followed the NIH Guide for the Care and Use of Laboratory Animals. The experimental protocol was approved by the local animal care committee. The hamster chamber window model is widely used for microvascular studies in the unanesthetized state, and the complete surgical technique is described in detail elsewhere ${ }^{14}$. Catheters were tunneled under the skin and exteriorized at the dorsal side of the neck, and securely attached to the window frame.

\section{Inclusion Criteria}

Animals were suitable for the experiments if: 1) systemic parameters were within normal range, namely, heart rate $(\mathrm{HR})>340$ beat $/ \mathrm{min}$, mean arterial blood pressure (MAP) $>80$ $\mathrm{mmHg}$, systemic Hct $>45 \%$, and arterial oxygen partial pressure $\left(\mathrm{PaO}_{2}\right)>50 \mathrm{mmHg}$; and 2) microscopic examination of the tissue in the chamber observed under a $\times 650$ magnification did not reveal signs of edema or bleeding.

\section{Acute hemorrhage protocol}

Acute hemorrhage was induced by withdrawing $50 \%$ of estimated total blood volume (BV) via the carotid artery catheter within $5 \mathrm{~min}$. Total BV was estimated as $7 \%$ of body weight. Animals were followed over $90 \mathrm{~min}$ after hemorrhage induction. The animals were categorized as non-survivors and euthanized earlier if at any time during the protocol their MAP fell below $30 \mathrm{mmHg}$ for more than 10 minutes.

\section{NO synthase during hemorrhagic shock}

NOS inhibition was induced by continuous infusion of L-NAME (N-nitro-L-arginine methyl ester, 30mg/kg, 10 $\mu 1 / \mathrm{min}$; Sigma-Aldrich. St. Louis, MO). Vehicle groups received saline at identical rate. L-NAME and vehicle infusion started $10 \mathrm{~min}$ after the end of the hemorrhage. Figure 1A presents the experimental protocol.

\section{Experimental groups for NO supplementation}

Nanoparticles were infused $10 \mathrm{~min}$ after the hemorrhage, suspended in 50 $\mu 1$ deoxygenated saline, and infused via the jugular vein $(100 \mu \mathrm{l} / \mathrm{min})$. Post-hemorrhage, animals were randomly divided ${ }^{15}$ into three groups: 1) NO-nps, $5 \mathrm{mg} / \mathrm{kg}$ of NO-nps; 2) Control-nps, 5 $\mathrm{mg} / \mathrm{kg}$ of NO free nanoparticles; 3) Vehicle, 50 $\mathrm{\mu l}$ deoxygenated saline. Eighteen $(\mathrm{n}=18)$ animals were entered into the study, and assigned to the following experimental groups: NO-nps $(n=6)$; Control-nps $(n=6)$; and Vehicle $(n=6)$.

\section{Systemic Parameters}

MAP and heart rate (HR) were recorded continuously (MP 150, Biopac System; Santa Barbara, CA). Hct was measured from centrifuged arterial blood samples taken in heparinized capillary tubes. Hb content was determined spectrophotometrically from a single drop of blood (Hemocue, Stockholm, Sweden). 


\section{Blood Chemistry and Biophysical Properties}

Arterial blood was collected in heparinized glass capillaries $(0.05 \mathrm{ml})$ and immediately analyzed for $\mathrm{PaO}_{2}, \mathrm{PaCO}_{2}$ and $\mathrm{pH}$ (Blood Chemistry Analyzer 248, Bayer, Norwood, MA). The comparatively low $\mathrm{PaO}_{2}$ and high $\mathrm{PaCO}_{2}$ of these animals is a consequence of their adaptation to a fossorial environment.

\section{Methemoglobin Measurement}

Methemoglobin was established according to Winterbourn ${ }^{16}$. Calibration is ensured using standard levels at 5.2\%, 2.6\% and 1.2\% MetHb (RNA Medical, Bayer Diagnostics Medfield, MA).

\section{Plasma nitrite/nitrate}

Blood samples were collected from carotid artery, and centrifuged to separate RBCs and plasma. Plasma proteins were removed by adding equal volume of methanol, and centrifuged at $15000 \mathrm{rpm}$ for $10 \mathrm{~min}$. Concentrations of NOx in the supernatant were measured with a NOx analyzer (ENO-20; Eicom, Kyoto, Japan). This analyzer combines Griess method and high-performance liquid chromatography.

\section{Microvascular Experimental Setup}

The unanesthetized animal was placed in a restraining tube with a longitudinal slit from which the window chamber protruded, then fixed to the microscopic stage of a transillumination intravital microscope (BX51WI, Olympus, New Hyde Park, NY). The animals were given $20 \mathrm{~min}$ to adjust to the change in the tube environment before measurements. The tissue image was projected onto a charge-coupled device camera (COHU 4815) connected to a videocassette recorder and viewed on a monitor. Measurements were carried out using a 40X (LUMPFL-WIR, numerical aperture 0.8, Olympus) water immersion objective. The same sites of study were followed throughout the experiment so that comparisons could be made directly to baseline levels.

\section{Microhemodynamics}

Arteriolar and venular blood flow velocities were measured on-line by using the photodiode cross-correlation method (Velocity Tracker Model 102B, Vista Electronics, San Diego, CA) ${ }^{17}$. Measured centerline velocity was corrected according to vessel size to obtain mean RBC velocity $(\mathrm{V})^{18}$. Video image-shearing method was used to measure vessel diameter (D) ${ }^{19}$. Blood flow (Q) was calculated from the measured values as $Q=\pi \times V(D / 2)^{2}$. This calculation assumes a parabolic velocity profile and has been found to be applicable to tubes of $15-80 \mu \mathrm{m}$ internal diameters and for Hcts in the range of $6-60 \%{ }^{18}$. Changes in arteriolar and venular diameter from baseline were used as indicators of a change in vascular tone. Peripheral vascular resistance (PVR) was calculated in terms of pressure (MAP) divide by local blood flow (Q), by mathematically using the Hagen-Poiseuille equation. PVR relative to baseline was calculated by ratio of MAP and Q normalized to baseline. Peripheral vascular hindrance was calculated by normalizing PVR by blood viscosity normalized to baseline, to eliminate viscous resistance component from the Poiseuille equation. Wall shear stress (WSS) was defined by WSS $=\mathrm{WSR} \times \eta$, where WSR is the wall shear rate given by $8 \mathrm{~V} \times \mathrm{D}^{-1}$, and $\eta$ is blood viscosity.

\section{Functional Capillary Density (FCD)}

Functional capillaries, defined as those capillary segments that have RBC transit of at least one $\mathrm{RBC}$ in a $45 \mathrm{~s}$ period in 10 successive microscopic fields were assessed, totaling a region of $0.46 \mathrm{~mm}^{2}$. FCD $\left(\mathrm{cm}^{-1}\right)$, i.e., total length of RBC perfused capillaries divided by the area of the microscopic field of view, was evaluated by measuring and adding the length of 
capillaries that had RBC transit in the field of view. Changes in FCD from baseline were used as indictors of capillary perfusion.

\section{Data analysis}

Results are presented as mean standard deviation. Data within each group were analyzed using analysis of variance for repeated measurements (ANOVA, Kruskal-Wallis test). When appropriate, post hoc analyses were performed with the Dunns multiple comparison test. Data between groups was analyzed using two-way ANOVA nonparametric repeated measurements, and, when appropriate, post hoc analyses were performed using Bonferroni tests. The Grubbs' method was used to assess closeness for all measured parameters values at baseline. Microhemodynamic measurements were compared to baseline levels obtained before the experimental procedure, for more robust statistics for small sample populations. All statistics were calculated using GraphPad Prism 4.01 (GraphPad Software, Inc., San Diego, CA). Changes were considered statistically significant if $\mathrm{P}<0.05$.

\section{RESULTS}

\section{NOS inhibition during hemorrhagic shock}

Twelve hamsters were used to establish effects of NO inhibition (L-NAME: $\mathrm{n}=6 ; 68.4 \pm 8.2$ g; and Vehicle: $\mathrm{n}=6 ; 66.2 \pm 7.1 \mathrm{~g}$ ). Hemorrhage significantly decreased Hct and $\mathrm{Hb}$ in both groups. Changes in MAP and HR post hemorrhage are shown in Figure 1B. MAP decreased after hemorrhage, and NOS inhibition produced an initial recovery in pressure, however some animals were not able to maintain the pressure. HR initially increased after hemorrhage, and NOS inhibition reduced HR compared to baseline and Vehicle. Diameters were constricted after hemorrhage, and NOS inhibition increased vasoconstriction compared to baseline and Vehicle. Microvascular flows and FCD were significantly decreased after hemorrhage compared to baseline in both groups. Peripheral vascular resistance is presented in Figure 1C. Two animals treated with L-NAME did not survive the experimental protocol (47 and 72 minutes), Figure 1D.

\section{NO supplementation during hemorrhagic shock}

A second group of animals was used to study the role of NO supplementation during hemorrhagic shock, divided in three groups: NO-nps: $(\mathrm{n}=6$; $66.2 \pm 5.1 \mathrm{~g})$; Control-nps: $(\mathrm{n}$ $=6 ; 63.8 \pm 4.4 \mathrm{~g})$; and Vehicle: $(\mathrm{n}=6 ; 66.1 \pm 4.8 \mathrm{~g})$. Figure 2A presents the experimental protocol. All animals tolerated the experimental protocol. All animals passed Grubbs' test ensuring that parameter at baseline were within a similar population.

\section{Blood gas chemistry}

Blood chemistry results are presented in Table 1. Hemorrhage decreased $\mathrm{Hct}$ and $\mathrm{Hb}$ in all groups. MetHb increased in animals treated with NO-nps compared to Control-nps and Vehicle. Plasma nitrite and nitrate decreased from baseline in animals that received the Vehicle or Control-nps, while animals treated with NO-nps presented and increased compared to baseline and to animals treated with Vehicle and Control-nps. Arterial blood $\mathrm{pO}_{2}$ after hemorrhage increased in all groups compared to baseline. However, animals treated with NO-nps had lower arterial $\mathrm{pO}_{2}$ compared to Vehicle and Control-nps. Arterial blood $\mathrm{pCO}_{2}$ after hemorrhage decreased in all groups compared to baseline, although, animals treated with NO-nps had higher arterial $\mathrm{pCO}_{2}$ compared to Vehicle and Control-nps. Arterial blood $\mathrm{pH}$ after hemorrhage decreased in all groups compared to baseline, and animals treated with NO-nps demonstrated a higher arterial $\mathrm{pH}$ as compared to Vehicle and Control-np. Blood and plasma viscosity were decreased compared to baseline in all groups. Plasma colloidal osmotic pressure (COP) decreased from baseline in all groups. 
Changes in MAP and HR post hemorrhage for all groups are shown in Figure 2 B, C. MAP in all groups decreased compared to baseline. Animals treated with NO-nps recovered MAP compared to vehicle within $30 \mathrm{~min}$ from the treatment, and after $90 \mathrm{~min}$ compared to Control-np. HR in all groups increased after hemorrhage, and after treatment HR dropped in animals treated with Control-nps and Vehicle. However, animals treated with NO-nps maintained HR over the observation time, and higher compared to animals treated with Control-nps and Vehicle.

Microvascular diameters and both arteriolar and venular blood flow are presented in Figure 3. Arteriolar and venular diameters (Figure $3 \mathrm{~A}, \mathrm{~B}$ ) were significantly constricted from baseline after hemorrhage for all groups. Microvascular flows were significantly decreased after hemorrhage compared to baseline for all groups. Arteriolar diameters remained constricted compared to baseline for Control-nps and Vehicle treated groups. Conversely, animals treated with NO-nps restored arteriolar diameters, and significant dilation was witnessed, compared to Control-nps and Vehicle treated groups. Arteriolar microvascular blood flow (Figure $3 \mathrm{C}, \mathrm{D}$ ) for all groups remained lower than baseline, although animals treated with NO-nps demonstrated higher arteriolar flow as compared to Control-nps and Vehicle treated animals. Venular diameters were constricted after hemorrhage for all groups compared to baseline, and remained constricted for Control-nps and Vehicle treated groups. Venular diameters in animals treated with NO-nps were not different from baseline. Venular blood flow decreased after hemorrhage compared to baseline, and remained lower than baseline for all groups. NO-nps had significantly higher venular blood flow as compared to Control-nps and Vehicle.

Changes in capillary perfusion during the protocol are presented in Figure 4 A. FCD was significantly reduced after hemorrhage for all groups, at all time points. FCD partially recovered in animals treated with NO-nps compared to Control-nps and Vehicle treated animals.

Estimated PVR are presented in Figure 4 B. PVR increased over the observation time for animals treated with Control-nps and Vehicle, significantly higher when compared to the NO-nps treated group. Calculated hemodynamic parameters are presented in Table 3. Peripheral vascular hindrance, which reflects the contribution of vascular geometry, was increased compared to baseline after hemorrhage independent of the treatment. Arteriolar and venular vessel WSR and WSS decreased after hemorrhage, compared to baseline, and remained decreased, independent of the treatment.

\section{DISCUSSION}

The principal finding of the study is that treatment of hemorrhagic shock by NO supplementation via NO-nps prevented cardiovascular collapse, and allowed the animals to maintain superior systemic and microvascular hemodynamic conditions as compared to controls. The importance of providing sustained exogenous NO after hemorrhage was evidenced in this study in the form of maintenance of heart rate, microvascular function, FCD and vascular resistance. Our findings relate to scenarios where an increase in NO bioavailability could potentially alter the course and ultimately survival, until volume resuscitation is available. Our results support the hypothesis that severe hemorrhage induces vascular decompensation associated with low $\mathrm{NO}$ availability, and that $\mathrm{NO}$ supplementation prevents the risks of profound hemorrhage. As generation of $\mathrm{NO}$ in vivo is closely linked to endothelial cell function, local shear stress, and local oxygen conditions, the lack of differences in WSS and Hct among groups, suggests that the differences observed between groups are mostly due to the NO released from NO-np. 
Hemorrhagic hypotension leads to a well-characterized sequence of events, and ultimately to vascular decompensation, due to a continuous increase in peripheral vascular resistance. Cardiovascular adaptation to hemorrhagic shock is dynamically controlled by endocrine and local paracrine factors, such as NO, in part to compensate for the sudden hypovolemia ${ }^{20}$. NO production keeps the vasculature relaxed, regulating blood pressure and tissue perfusion. The effects of perfusion on gas exchange are one of the most important features of NO supplementation in pulmonary physiology. As changes in the distribution of blood flow to different areas of the lung, and adjustments of vessel diameter in the respective regions of the lung, could rapidly affect gas exchange. NO is a key molecule for fast response, which links alveolar ventilation to local lung perfusion. Hamster's environmental adaptations are responsible for their low arterial $\mathrm{PO}_{2}$, their natural environment are in burrows, either permanently or intermittently, and often demonstrate structural and functional differences from surface dwelling mammals. The marked improvement in gas exchange, decrease in oxygen content and increase in $\mathrm{PCO}_{2}$ seen in the NO-group can be the result of improved ventilation-perfusion, as $\mathrm{NO}$ has the singular property of preferentially dilating vascular segments located in ventilated areas ${ }^{21}$. Additionally, NO modulates synaptic signaling, cellular defense and mitochondria oxygen utilization ${ }^{22}$. Our results indicate that restoring intravascular NO concentrations by replenishing/increasing intravascular NO availability by treatment with NO-nps reduces vascular complications resulting from hemorrhage shock. The exogenous NO released induced by treatment with $5 \mathrm{mg} / \mathrm{kg}$ of NO-nps was evidenced by significant vasodilation, metHb formation, and increase in plasma nitrite and nitrate.

In our study, NOS inhibition caused an increase of vascular resistance, decreased heat rate, and increased mortality. Similarly, a clinical study using L-NAME was discontinued because the treatment showed higher mortality ${ }^{23}$. Other NOS inhibitors were clinically evaluated to prevent hypotension; with poor results, as they increased ischemia and cardiovascular collapse 24,25 . Several investigations suggest that inducible NOS (iNOS) is responsible in part for producing organ injury after hemorrhage and resuscitation ${ }^{26-28}$. Until selective iNOS inhibitors are available, these findings should be analyzed with discretion. Moreover, hypoxia and ischemia during shock initiate tissue adaptive responses through transcriptional activation of several factors, such as HIF-1, which requires NO as a regulatory molecule. Traditionally, NO has been thought to be a signal, exclusively via its stimulation of guanylyl cyclase, inducing an increase in intracellular cGMP levels and, in turn, the allosteric activation of cGMP dependent kinase. Moreover, in addition to the activation of PKG, the cGMP-dependent effects can also be mediated by other proteins whose activities are allosterically modified by cGMP, and via cross-activation of cAMPdependent kinase. In previous work, we have demonstrated that NO-np vascular effect effects are mediated by guanylyl cyclase, and can be enhanced by phosphodiesterase inhibitors and blocked by irreversible heme-site inhibitors of soluble guanylyl cyclase ${ }^{13}$. Thus, acute inhibition of phosphodiesterase activity, by drugs such as sildenafil, combined with NO-np represents an attractive therapeutic strategy to manipulate systemic vascular tone.

Exogenous NO in the form of NO-nps treatment following severe hemorrhag, partially recovered systemic and microvascular conditions, via reduction of precapillary resistance when compared to animals treated with Control-nps or the Vehicle. Results show a strong correlation between cardiac stability and microvascular flow. Mechanistically, it is expected that intravascular NO supplementation in the form of NO-np reduces MAP by decreasing PVR. Conversely, the experimental results indicate that NO-np increased sustained higher MAP and HR during the observation period. Central cardiac effects could reflect increases in cardiac output mediated by lower arteriolar after-load, cardiac filling and increased preload (venous return), due to NO vasodilatory properties and cardiac chronotropic effects ${ }^{28}$. NO is a regulator of cardiac function through indirect vascular-dependent 
mechanisms and by direct action on the myocardium as a paracrine autacoid involved in autonomic control and contractility ${ }^{29}$. This effect appears to be NO concentrations dependent, as physiological levels of NO increases HR via activation of hyperpolarization induced inward current, and is less pronounced at high concentrations of $\mathrm{NO}^{30,31}$. The exogenous NO released from the NO-nps maintained central hemodynamic function by preventing rhythm disturbances, implying a protective role of $\mathrm{NO}$ on cardiac over-drive and pacing ${ }^{32}$. NO has effects in cardiomyocytes, which are cyclic GMP-dependent and other effects that are cyclic GMP-independent ${ }^{34}$. Heart muscle cells express the soluble isoform of guanylyl cyclase that catalytically increases intracellular levels of cyclic GMP in response to stimulation by pure $\mathrm{NO}$, or GSNO ${ }^{34}$. Moreover, heart rate can directly affect cardiac output, if stroke volume remains constant, therefore results observed with NO-np can be the result of sustaining higher heart rates and potentially, cardiac output, which ultimately increase systemic oxygen delivery. These results show that the principal factor in ensuring hemodynamic restoration by NO-nps is not related to a volume effect since hematocrits did not differ between groups.

Clinically, vasodilators as adjunct therapy to fluid resuscitation were shown to be effective in restoring the microvascular function ${ }^{35}$. Nitroglycerin with adequate volume support, has been shown to reinstate capillary flow and restore sublingual microcirculation ${ }^{36}$. This work explores the connection between cardiovascular collapse post hemorrhagic shock, and reduced NO bioavailability, by increasing the NO bound to low molecular weight thiols (1 $\mathrm{mg} / \mathrm{kg}$ nitrosoglutathione, GSNO) prior to the hemorrhage ${ }^{36}$. Since GSNO only is stable in vivo at basal conditions for a limited period of time, its application is limited. Therefore, our current study goes beyond previous results with GSNO administrated prior hemorrhage, to a therapy applicable $10 \mathrm{~min}$ post the hemorrhage based on NO-np. The most important characteristic to supplement $\mathrm{NO}$ post hemorrhage is the intravascular controlled release of moderate levels of NO to mimic physiologically generated NO, a characteristic currently achievable with NO-np. NO donors, such as nitroglycerin, an organic nitrate, and clinically approved, is short acting, involves specific enzymes and reducing agents, and it is not suitable for a controlled and sustained release. Other NO donors, where the NO is bound to a nucleophile, and the NO is liberated after spontaneous decomposition, were not suitable as therapies post hemorrhage. NO-nanoparticles release pure NO, freely soluble in physiological solution, and do not require cofactors to facilitate NO release. The current results indicate that treatment with $5 \mathrm{mg} / \mathrm{kg} \mathrm{NO}$-nps, prevented hemorrhagic shock, induced vascular decompensation, and set the proof of the concept that $\mathrm{NO}$ supplementation after hemorrhage will reduce the risks of cardiovascular collapse. To date, there are no definitive treatments for addressing massive blood loss on the battlefield, and existing therapies for severe hemorrhage are particularly limited. NO-nps treatment maintains perfusion reducing hemorrhagic shock sequelae, with an infusion volume of less than $2.5 \%$ of the shed blood volume. NO-np is the logical progression of the concepts initially defined with GSNO, as GSNO is compromised by thermal decomposition, photolysis and in vivo catalytic decomposition. The reduction in weight therapy provided by NO-np relative to fluid resuscitation can greatly reduce logistical burdens, and the stability of NO-nps for months exposed to air at room temperature, facilitates its application in multiple scenarios.

In conclusion, this study shows that severe hemorrhage induces vascular decompensation, in part, due to low availability of NO during post hemorrhage, and that exogenous NO during this stage can prevent circulatory arrest. Exogenous NO in the from of NO-nps attenuated microvascular complications during hypovolemia, which targets a pivotal protective function by maintaining tissue perfusion, assuring wash-out of metabolic residues and thereby preventing future damage during reperfusion. Mechanistically, partial preservation of microvascular perfusion during hypovolemic shock due to increased NO bioavailability, acts by regulating vascular tone and pressure redistribution, and maintaining capillary 
pressure and metabolite exchange. Lastly, NO has a fundamental signaling role in cellular function with implications in cardiac chronotropic function, which appears to be jeopardized as hypovolemic shock is established.

\section{Acknowledgments}

This work was supported by Bioengineering Research Partnership grant R24-HL64395, grant R01-HL62354, the FJC, A Foundation of Philanthropic Funds. AJF was supported by research grants from the American Society for Dermatologic Surgery Cutting Edge Program, the La-Roche Posay North American Foundation, and the Women's Dermatologic Society. The authors thank Froilan P. Barra and Cynthia Walser for the surgical preparation of the animals.

\section{REFERENCES}

1. Bellamy RF. The causes of death in conventional land warfare: implications for combat casualty care research. Mil Med. 1984; 149:55-62. [PubMed: 6427656]

2. Pope, AM. Institute of Medicine (U.S.). Fluid resuscitation : state of the science for treating combat casualties and civilian injuries. Washington, D.C.: National Academy Press; 1999.

3. Bacter CR, Canizaro PC, Carrico CJ, Shires GT. Fluid resuscitation of hemorrhagic shock. Postgrad Med. 1970; 48:95-99. [PubMed: 5460379]

4. Burris D, Rhee P, Kaufmann C, Pikoulis E, Austin B, Eror A, et al. Controlled resuscitation for uncontrolled hemorrhagic shock. J Trauma. 1999; 46:216-223. [PubMed: 10029024]

5. Dubick MA, Atkins JL. Small-volume fluid resuscitation for the far-forward combat environment: current concepts. J Trauma. 2003; 54:S43-S45. [PubMed: 12768102]

6. Kristiansson M, Soop M, Saraste L, Sundqvist KG. Cytokines in stored red blood cell concentrates: promoters of systemic inflammation and simulators of acute transfusion reactions? Acta Anaesthesiol Scand. 1996; 40:496-501. [PubMed: 8738697]

7. Ignarro LJ, Byrns RE, Buga GM, Wood KS. Endothelium-derived relaxing factor from pulmonary artery and vein possesses pharmacologic and chemical properties identical to those of nitric oxide radical. Circ Res. 1987; 61:866-879. [PubMed: 2890446]

8. Salazar Vazquez BY, Martini J, Chavez Negrete A, Cabrales P, Tsai AG, Intaglietta M. Microvascular benefits of increasing plasma viscosity and maintaining blood viscosity: counterintuitive experimental findings. Biorheology. 2009; 46:167-179. [PubMed: 19581725]

9. Bevers LM, Braam B, Post JA, van Zonneveld AJ, Rabelink TJ, Koomans HA, et al. Tetrahydrobiopterin, but not L-arginine, decreases NO synthase uncoupling in cells expressing high levels of endothelial NO synthase. Hypertension. 2006; 47:87-94. [PubMed: 16344367]

10. Allen BW, Demchenko IT, Piantadosi CA. Two faces of nitric oxide: implications for cellular mechanisms of oxygen toxicity. J Appl Physiol. 2009; 106:662-667. [PubMed: 18845774]

11. Lancaster JR. Simulation of the diffusion and reaction of endogenously produced nitric oxide. Proc Natl Acad Sci USA. 1994; 91:8137-8141. [PubMed: 8058769]

12. Friedman AJ, Han G, Navati MS, Chacko M, Gunther L, Alfieri A, et al. Sustained release nitric oxide releasing nanoparticles: characterization of a novel delivery platform based on nitrite containing hydrogel/glass composites. Nitric Oxide. 2008; 19:12-20. [PubMed: 18457680]

13. Cabrales P, Han G, Roche C, Nacharaju P, Friedman AJ, Friedman JM. Sustained release nitric oxide from long-lived circulating nanoparticles. Free Radic Biol Med. 2010

14. Colantuoni A, Bertuglia S, Intaglietta M. Quantitation of rhythmic diameter changes in arterial microcirculation. Am J Physiol. 1984; 246:H508-H517. [PubMed: 6720909]

15. Altman DG, Bland JM. Statistics notes: How to randomise. BMJ. 1999; 319:703-704. [PubMed: 10480833]

16. Winterbourn, CC. Reaction of superoxide with hemoglobin. CRC Handbook of Methods for Oxygen Radical Research. Boca Raton, Florida: CRC Press; 1985.

17. Intaglietta M, Silverman NR, Tompkins WR. Capillary flow velocity measurements in vivo and in situ by television methods. Microvasc Res. 1975; 10:165-179. [PubMed: 1186524] 
18. Lipowsky HH, Zweifach BW. Application of the "two-slit" photometric technique to the measurement of microvascular volumetric flow rates. Microvasc Res. 1978; 15:93-101. [PubMed: 634160]

19. Intaglietta M, Tompkins WR. Microvascular measurements by video image shearing and splitting. Microvasc Res. 1973; 5:309-312. [PubMed: 4709728]

20. Schadt JC, Ludbrook J. Hemodynamic and neurohumoral responses to acute hypovolemia in conscious mammals. Am J Physiol. 1991; 260:H305-H318. [PubMed: 1671735]

21. Rossaint R, Falke KJ, Lopez F, Slama K, Pison U, Zapol WM. Inhaled nitric oxide for the adult respiratory distress syndrome. N Engl J Med. 1993; 328:399-405. [PubMed: 8357359]

22. Moncada S, Erusalimsky JD. Does nitric oxide modulate mitochondrial energy generation and apoptosis? Nat Rev Mol Cell Biol. 2002; 3:214-220. [PubMed: 11994742]

23. Lopez A, Lorente JA, Steingrub J, Bakker J, McLuckie A, Willatts S, et al. Multiple-center, randomized, placebo-controlled, double-blind study of the nitric oxide synthase inhibitor 546C88: effect on survival in patients with septic shock. Crit Care Med. 2004; 32:21-30. [PubMed: 14707556]

24. Md S, Moochhala SM, Siew-Yang KL. The role of inducible nitric oxide synthase inhibitor on the arteriolar hyporesponsiveness in hemorrhagic-shocked rats. Life Sci. 2003; 73:1825-1834. [PubMed: 12888121]

25. Thiemermann C, Szabo C, Mitchell JA, Vane JR. Vascular hyporeactivity to vasoconstrictor agents and hemodynamic decompensation in hemorrhagic shock is mediated by nitric oxide. Proc Natl Acad Sci U S A. 1993; 90:267-271. [PubMed: 7678341]

26. Banta S, Yokoyama T, Berthiaume F, Yarmush ML. Effects of dehydroepiandrosterone administration on rat hepatic metabolism following thermal injury. J Surg Res. 2005; 127:93-105. [PubMed: 15882877]

27. Shimizu T, Tani T, Endo Y, Hanasawa K, Tsuchiya M, Kodama M. Elevation of plasma peptidoglycan and peripheral blood neutrophil activation during hemorrhagic shock: plasma peptidoglycan reflects bacterial translocation and may affect neutrophil activation. Crit Care Med. 2002; 30:77-82. [PubMed: 11902292]

28. Menezes JM, Hierholzer C, Watkins SC, Billiar TR, Peitzman AB, Harbrecht BG. The modulation of hepatic injury and heat shock expression by inhibition of inducible nitric oxide synthase after hemorrhagic shock. Shock. 2002; 17:13-18. [PubMed: 11795663]

29. Fatehi-Hassanabad Z, Fatehi M. Characterisation of some pharmacological effects of the venom from Vipera lebetina. Toxicon. 2004; 43:385-391. [PubMed: 15051401]

30. Han X, Kobzik L, Zhao YY, Opel DJ, Liu WD, Kelly RA, et al. Nitric oxide regulation of atrioventricular node excitability. Can J Cardiol. 1997; 13:1191-1201. [PubMed: 9444302]

31. Herring N, Rigg L, Terrar DA, Paterson DJ. NO-cGMP pathway increases the hyperpolarisationactivated current, I(f), and heart rate during adrenergic stimulation. Cardiovasc Res. 2001; 52:446453. [PubMed: 11738061]

32. Massion PB, Balligand JL. Modulation of cardiac contraction, relaxation and rate by the endothelial nitric oxide synthase (eNOS): lessons from genetically modified mice. J Physiol. 2003; 546:63-75. [PubMed: 12509479]

33. Khadour FH, O'Brien DW, Fu Y, Armstrong PW, Schulz R. Endothelial nitric oxide synthase increases in left atria of dogs with pacing-induced heart failure. Am J Physiol. 1998; 275:H1971H1978. [PubMed: 9843795]

34. Mayer B, Pfeiffer S, Schrammel A, Koesling D, Schmidt K, Brunner F. A new pathway of nitric oxide/cyclic GMP signaling involving S-nitrosoglutathione. J Biol Chem. 1998; 273:3264-3270. [PubMed: 9452441]

35. De Backer D, Creteur J, Dubois MJ, Sakr Y, Vincent JL. Microvascular alterations in patients with acute severe heart failure and cardiogenic shock. Am Heart J. 2004; 147:91-99. [PubMed: 14691425]

36. Spronk PE, Ince C, Gardien MJ, Mathura KR, Oudemans-van Straaten HM, Zandstra DF. Nitroglycerin in septic shock after intravascular volume resuscitation. Lancet. 2002; 360:13951396. [PubMed: 12423989] 
37. Cabrales P, Tsai AG, Intaglietta M. Exogenous nitric oxide induces protection during hemorrhagic shock. Resuscitation. 2009; 80:707-712. [PubMed: 19362408] 


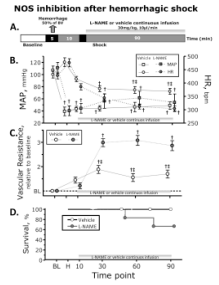

Figure 1.

Nitric oxide (NO) synthase (NOS) inhibition during hemorrhagic shock. A. Shock protocol. Hemorrhagic shock was induced withdrawal of $50 \%$ of the estimated blood volume $(7 \%$ body weight) and NOS inhibition with L-NAME was started $10 \mathrm{~min}$ after the end of the hemorrhage. B. NOS inhibition effects on mean arterial pressure (MAP) and heart rate (HR) during the hemorrhagic shock. C. NOS inhibition effects on vascular resistance during the hemorrhagic shock. D. NOS inhibition effects on survivability during the hemorrhagic shock. $\dagger, \mathrm{P}<0.05$ compared to baseline; $\ddagger, \mathrm{P}<0.05$ compared to Vehicle 


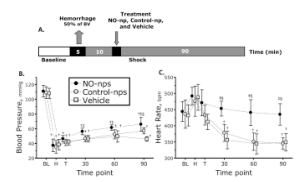

Figure 2.

A. Diagram hemorrhagic shock protocol. Hemorrhagic shock was induced withdrawal of $50 \%$ of the estimated blood volume ( $7 \%$ body weight). Treatments were administered 10 min after the end of the hemorrhage. B. and C. nitric oxide (NO) supplementation effects on mean arterial pressure (MAP) and heart rate (HR) during hemorrhagic shock. $\dagger, \mathrm{P}<0.05$ compared to baseline; $\ddagger, \mathrm{P}<0.05$ compared to Vehicle; $\S, \mathrm{P}<0.05$ compared to Control-nps. Time points: Bl, baseline; $\mathrm{H}$, after hemorrhage, $\mathrm{T}$, after treatment, and 30, 60 and $90 \mathrm{~min}$ after treatment. MAP $(\mathrm{mmHg}$, mean $\pm \mathrm{SD})$ for each animal group were as follows: Baseline: Vehicle, $108 \pm 7, n=6$; Control-nps, $109 \pm 8 n=6$; NO-nps, $111 \pm 8, n=6 . n=$ number of animals. HR (bpm, mean \pm SD) at baseline for each animal group was as follows: Vehicle, $432 \pm 32$; Control-nps, $436 \pm 43 ;$ NO-nps, $444 \pm 31$. 


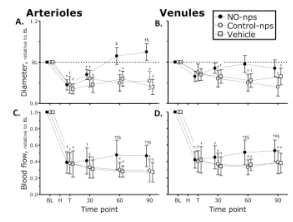

Figure 3.

Nitric oxide (NO) supplementation effects on changes in arteriolar and venular diameter (A. and B.) and blood flow (C. and D.) during hemorrhagic shock. Broken line represents baseline level. Time points: Bl, baseline; H, after hemorrhage, T, after treatment, and 30, 60 and 90 min after treatment. $\dagger, \mathrm{P}<0.05$ compared to baseline; $\ddagger, \mathrm{P}<0.05$ compared to Vehicle; $\S, \mathrm{P}<0.05$ compared to Control-nps. Diameters ( $\mu \mathrm{m}$, mean $\pm \mathrm{SD}$ ) in Figures 3A (arteriolar) and 3B (venular) for each animal group were as follows: Baseline: Vehicle (arterioles (A): $62.7 \pm 8.2, \mathrm{n}=26$; venules (V): $64.5 \pm 6.8, \mathrm{n}=24)$; Control-nps (A: $60.5 \pm 6.8, \mathrm{n}=24$; V: $65.7 \pm 8.7, \mathrm{n}=27) ; \boldsymbol{N O}$-nps (A: $62.0 \pm 7.4, \mathrm{n}=25, \mathrm{~V}: 64.5 \pm 8.2, \mathrm{n}=26) . \mathrm{n}=$ number of vessels studied. RBC velocities ( $\mathrm{mm} / \mathrm{s}$, mean $\pm \mathrm{SD}$ for each animal group were as follows: Baseline: Vehicle (A: $4.3 \pm 1.0, \mathrm{~V}: 2.3 \pm 0.9$ ); Control-nps (A: $4.5 \pm 0.8$; V: $2.4 \pm 1.0$ ); NOnps (A: $4.3 \pm 1.0 ; \mathrm{V}: 2.6 \pm 0.7)$. Calculated flows $(\mathrm{nl} / \mathrm{s}$, mean $\pm \mathrm{SD}$ ) in Figures 3C (arteriolar) and 3D (venular) for each animal group were as follows: Baseline: Vehicle (A: $11.7 \pm 3.4 ; \mathrm{V}: 6.9 \pm 2.2$ ); Control-nps (A: $12.1 \pm 3.2 ; \mathrm{V}: 7.1 \pm 2.3$ ); NO-nps (A: $12.0 \pm 2.8$; $\mathrm{V}: 6.8 \pm 2.3)$. 

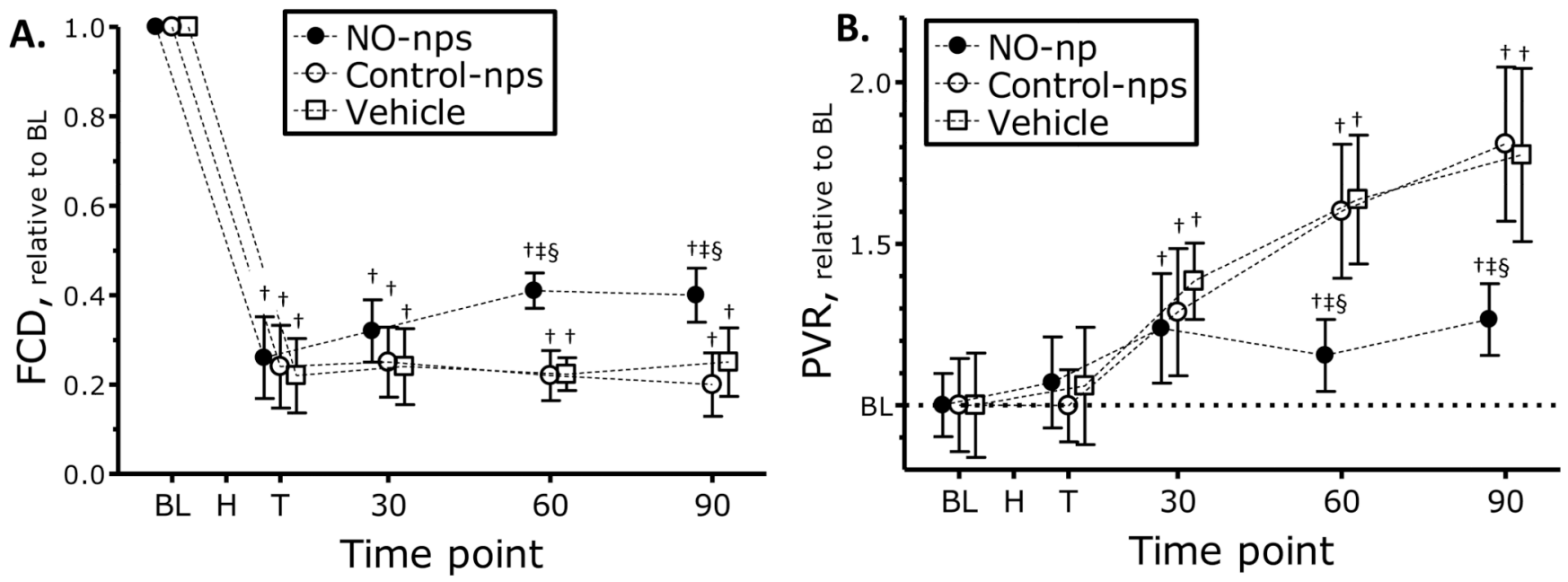

Figure 4.

A. Nitric oxide (NO) supplementation effects on changes in functional capillary density (FCD) and B. estimated peripheral vascular resistance (PVR) during hemorrhagic shock. Broken line represents baseline level. Estimation of PVR was made using Hagen-Poiseuille equation, with MAP and microvascular blood flow. Time points: Bl, baseline; H, after hemorrhage, T, after treatment, and 30, 60 and 90 min after treatment. $\dagger, \mathrm{P}<0.05$ compared to baseline; $\ddagger, \mathrm{P}<0.05$ compared to Vehicle; $\S, \mathrm{P}<0.05$ compared to Control-nps. FCD (capillaries per $\mathrm{cm}$, mean $\pm \mathrm{SD}$ ) for each animal group were as follows: Baseline: Vehicle, $111 \pm 9 ;$ Control-nps, $112 \pm 11 ;$ NO-nps, $109 \pm 8$. 


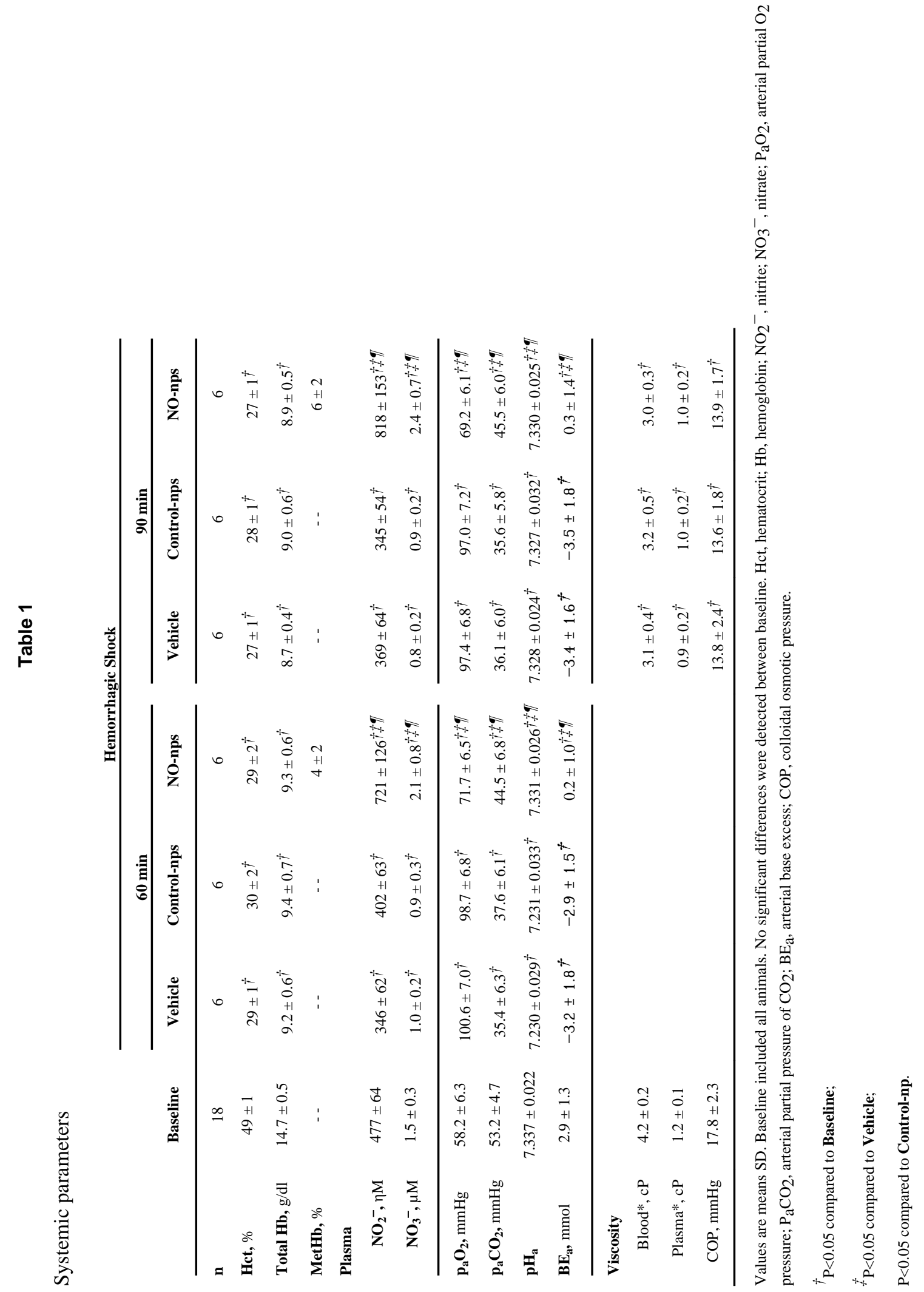


Table 2

Estimated vascular resistance and wall shear rate and stress

\begin{tabular}{lcccc} 
& & \multicolumn{3}{c}{ Hemorrhagic Shock 90 min } \\
\cline { 3 - 5 } & Baseline & Vehicle & Control-nps & NO-nps \\
\hline $\begin{array}{c}\text { Vascular resistance, } \\
\text { relative to baseline }\end{array}$ & 1.0 & $1.8 \pm 0.3^{\dagger}$ & $1.7 \pm 0.4^{\dagger}$ & $1.3 \pm 0.3^{\dagger}$ \\
\hline $\begin{array}{l}\text { Vascular hindrance } \\
\text { relative to baseline }\end{array}$ & 1.0 & $2.5 \pm 0.5^{\dagger}$ & $2.5 \pm 0.6^{\dagger}$ & $1.7 \pm 0.5^{\dagger}$ \\
\hline Arteriolar & & & & \\
$\begin{array}{l}\text { WSR, s-1 } \\
\text { WSS, dyn. cm-2 }\end{array}$ & $306 \pm 92$ & $258 \pm 57^{\dagger}$ & $254 \pm 52^{\dagger}$ & $292 \pm 64^{\dagger}$ \\
& & $8 \pm 2^{\dagger}$ & $8 \pm 2^{\dagger}$ & $9 \pm 2^{\dagger}$ \\
Venular & & & \\
WSR, s-1 & $456 \pm 84$ & $196 \pm 48^{\dagger}$ & $244 \pm 51^{\dagger}$ & $268 \pm 46^{\dagger}$ \\
WSS, dyn. cm-2 & $20 \pm 4$ & $7 \pm 2^{\dagger}$ & $8 \pm 2^{\dagger}$ & $8 \pm 2^{\dagger}$ \\
\hline
\end{tabular}

Hematocrits are presented in Table 1. WSR, wall shear rate; WSS, wall shear stress.

${ }^{\dagger} \mathrm{P}<0.05$ compared to Baseline;

${ }^{\dagger}<0.05$ compared to Vehicle;

$\mathrm{P}<0.05$ compared to Control-np. 